\title{
Publisher Correction: The chemical basis of ferroptosis
}

\section{Marcus Conrad (D) and Derek A. Pratt (1D)}

Correction to: Nature Chemical Biology https://doi.org/10.1038/s41589-019-0408-1, published online 18 November 2019

In the version of this article previously published, several components of Fig. 1 were missing. In panels $\mathbf{c}$ and $\mathbf{d}$, radicals were omitted from the compound structures; in panel e, the arrow going downward from arachidonate was partially removed. Additionally, the structure of ML094 in Fig. 4 was incorrect. The 1,3-cyclohexadiene ring on the south end of the molecule should be a naphthyl ring. The errors have been corrected in the HTML and PDF versions of the paper.

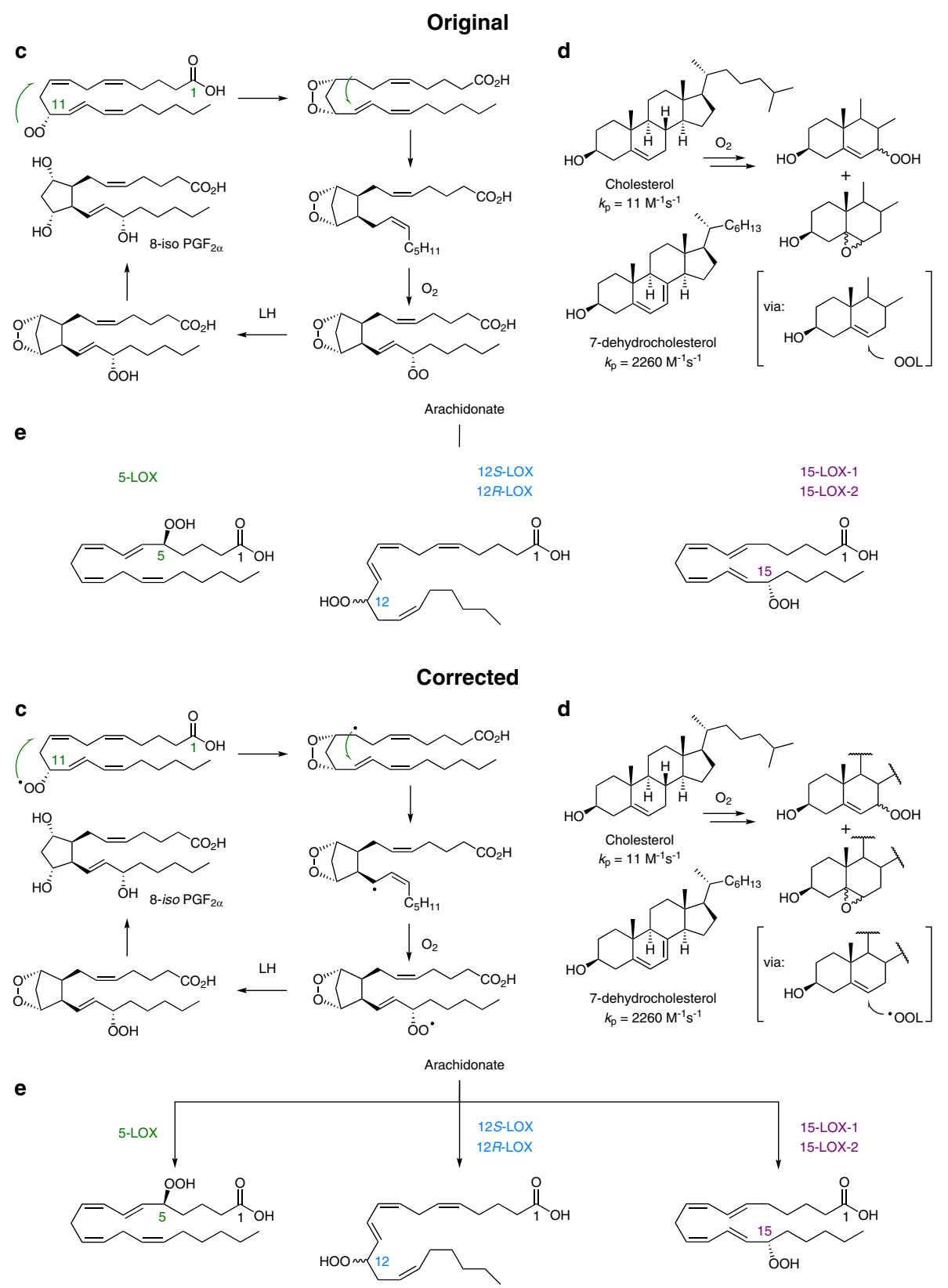

Fig. 1 | Original and Corrected. 
Original

Redox active inhibitors<smiles>CC(Cc1ccc(O)c(O)c1)C(C)Cc1ccc(O)c(O)c1</smiles><smiles>CC(c1cc2ccccc2s1)N(O)C(N)=O</smiles><smiles>O=c1cc(-c2ccccc2)oc2cc(O)c(O)c(O)c12</smiles><smiles>c1ccc2c(c1)SCc1c-2[nH]c2ccccc12</smiles>

Non-selective

LOX inhibitor

Zileuton 5-LOX inhibitor

Baicalein 12S-LOX inhibitor

PD146176

$k_{\text {inh }}=6.1 \times 10^{3}$

$K_{\text {inh }}=3.2 \times 10^{4}$ 15-LOX-1 inhibitor

$k_{\text {inh }}=6.6 \times 10^{3}$<smiles>N#C/C(=C\c1ccc(O)c(O)c1)C(=O)OC/C=C/c1ccccc1</smiles>

Redox inactive inhibitors

CDC<smiles>COc1ccc(/C=C2/SC(c3ccc(Cl)cc3)=NC2=O)cc1</smiles>

Non-selective

LOX inhibitor

$k_{\text {inh }}=1.1 \times 10^{4}$<smiles>Cc1nccn1-c1ccc(Sc2cccc(C3(C(N)=O)CCOCC3)c2)cc1</smiles>

CJ13610 5-LOX inhibitor

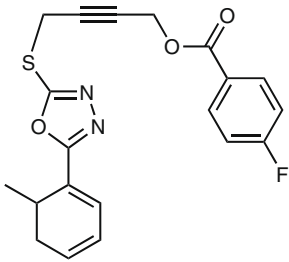

ML-094 15-LOX-1 inhibitor

Corrected

Redox active inhibitors<smiles>CC(Cc1ccc(O)c(O)c1)C(C)Cc1ccc(O)c(O)c1</smiles><smiles>O=c1cc(-c2ccccc2)oc2cc(O)c(O)c(O)c12</smiles>

Baicalein 12S-LOX inhibitor

$k_{\text {inh }}=3.2 \times 10^{4}$<smiles>c1ccc2c(c1)CSc1c-2[nH]c2ccccc12</smiles>

PD146176 15-LOX-1 inhibitor $k_{\text {inh }}=6.6 \times 10^{3}$<smiles>N#C/C(=C\c1ccc(O)c(O)c1)C(=O)OC/C=C/c1ccccc1</smiles>

Redox inactive inhibitors<smiles>COc1ccc(/C=C2/SC(c3ccc(Cl)cc3)=NC2=O)cc1</smiles>

$\mathrm{CDC}$
Non-selective

Non-selective

LOX inhibitor

$k_{\text {inh }}=1.1 \times 10^{4}$

CAY10649

5-LOX inhibito<smiles>Cc1nccn1-c1ccc(Sc2cccc(C3(C(N)=O)CCOCC3)c2)cc1</smiles>

CJ13610

5-LOX inhibitor



Fig. 4 | Original and Corrected.

Published online: 2 January 2020

https://doi.org/10.1038/s41589-019-0434-z

(๑) The Author(s), under exclusive licence to Springer Nature America, Inc. 2020 